\title{
Folded Compact Ultra-Wideband Stepped-Impedance Resonator Filters
}

\author{
Marjan Mokhtaari ${ }^{1}$, Jens Bornemann ${ }^{1}$ and Smain Amari ${ }^{2}$ \\ ${ }^{1}$ University of Victoria, Victoria, BC, Canada V8W 3P6 \\ ${ }^{2}$ Royal Military College of Canada, Kingston, ON, Canada K7K 7B4
}

\begin{abstract}
New ultra-wideband stepped-impedance resonator configurations are presented which, in cascaded and folded forms, are well suited for compact small-size filter applications. Compared to similar structures known hitherto, improved stopband performance is obtained by structural folding and source-load coupling. A variety of different filter configurations is presented with bandwidths up to $\mathbf{1 0 5}$ percent covering the 3.110.6 GHz range. Excellent stopband performance is obtained up to $16 \mathrm{GHz}$. The filters are designed for standard microstrip applications on commonly used substrate materials, and their responses are verified by different commercial electromagnetic software packages.
\end{abstract}

Index Terms - Filter design, ultra-wideband filters, stepped impedance resonators, microstrip filters.

\section{INTRODUCTION}

The tunable harmonic response property of steppedimpedance resonators (SIR's) is well known and has been extensively applied to the design of dual-band printed-circuit filters, e.g., [1] - [8].

Ultra-wideband (UWB) filters are often designed as microstrip circuits including additional apertures in the ground plane, e.g. [9], [10], or in coplanar waveguide technology [11]. The multiple resonances of a single stepped-impedance resonator (Fig. 1) are employed in [12], [13] and, using additional stubs and source-load coupling, in [14]. Cascaded stepped-impedance filters for UWB applications are presented in [15].

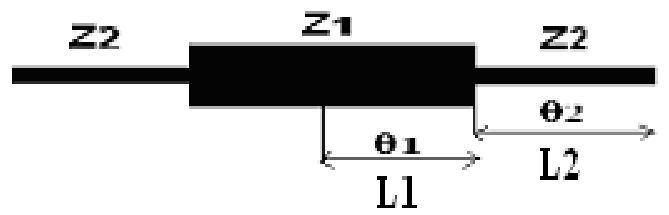

Fig. 1. Electrical and dimensional parameters of a steppedimpedance resonator (SIR).

From all of these investigations (and many others not referenced here for lack of space), it is obvious that a single stepped-impedance resonator, which is tightly coupled to the input/output microstrip lines, is capable of producing an UWB filter covering the 3.1-10.6 GHz frequency range, e.g. [13]. However, the stopband behavior of such a filter is rather poor. On the other hand, cascading multiple stepped-impedance resonators provide excellent UWB filters with good stopband performance; but the designs are rather long and spacious [15].
Therefore, this paper focuses on structural folding of both the stepped-impedance resonators (Fig. 2) and the entire component to obtain compact UWB filters with improved stopband performance. As a consequence of folding, sourceload (input-output) coupling can easily be applied to introduce transmission zeros in the stopband.

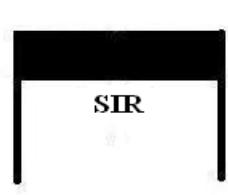

(a)

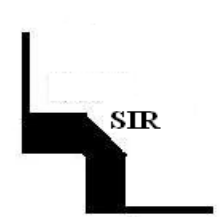

(b)

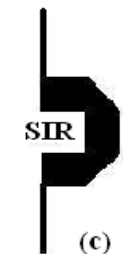

(c)
Fig. 2. Examples of folded SIR's.

\section{DESIGN}

Fig. 1 depicts the geometry of a microstrip SIR. The resonant frequencies are calculated by the following two transcendental equations:

$$
\begin{array}{ll}
R_{z}-\tan \theta_{1} \tan \theta_{2}=0 & \text { odd mode } \\
R_{z} \tan \theta_{1}+\tan \theta_{2}=0 & \text { even mode }
\end{array}
$$

where $R_{z}=Z_{2} / Z_{1}$ is the impedance ratio and $\theta_{1}$ and $\theta_{2}$ are the electrical lengths of the respective impedance segments. Fundamental and higher-order odd and even resonances occur alternatively. The ratio of the higher resonance frequencies to the fundamental frequency can be determined through above equations in terms of $R_{z}$ and the ratio $U$ of the electrical lengths.

$$
U=\theta_{2} /\left(\theta_{1}+\theta_{2}\right)
$$

Note that the ratio of the physical lengths differs from that of the electrical lengths because the effective dielectric constants are different for the two lines.

In order to design a wide band filter, the fundamental and the proper number of higher order resonances of the SIR should be located within the passband. For triple-mode SIR's, the center frequency of the filter is the arithmetic mean of the fundamental and second resonant frequencies so that the fundamental and second harmonic frequencies are properly defined close to the edges of the passband and equally spaced from the center frequency.

The SIR's are connected to each other and to the input/output by quarter-wavelength coupled-line sections, which act as inverters and can generate two more reflection zeros within the passband, e.g. [13]. 
The concept of UWB filter design is to match the resonant frequencies of the SIR's with the reflection zeros generated from a Chebyshev polynomial [15]. High selectivity is achieved by allocating transmission zeros close to the passband. In the folded configurations presented here, the value and sign of the source-load coupling defines the locations of the transmission zeros. A strong and positive source-load coupling can generate two transmission zeros on each side of the passband and a number of other zeros in the upper stopband region.

\section{RESULTS}

This section presents design examples of folded UWB filters using one, two and three SIR's. All designs are verified using the two commercial software packages Ansoft Designer $^{\circledR}$ and IE3D ${ }^{\circledR}$.

Fig. 3 shows the responses of two UWB filters using the folded SIR configurations in Figs. $2 \mathrm{~b}$ and 2c, respectively. The substrate is RT6010 with $\varepsilon_{\mathrm{r}}=10.8$ and a thickness of 50 mils. The fractional 3-dB bandwidths for these filters are 91.6 and 93.6 percent, respectively. It appears that the folded SIR in Fig. 3c shows slightly higher fractional bandwidth with the same structural parameters. The insertion loss is less than $2 \mathrm{~dB}$
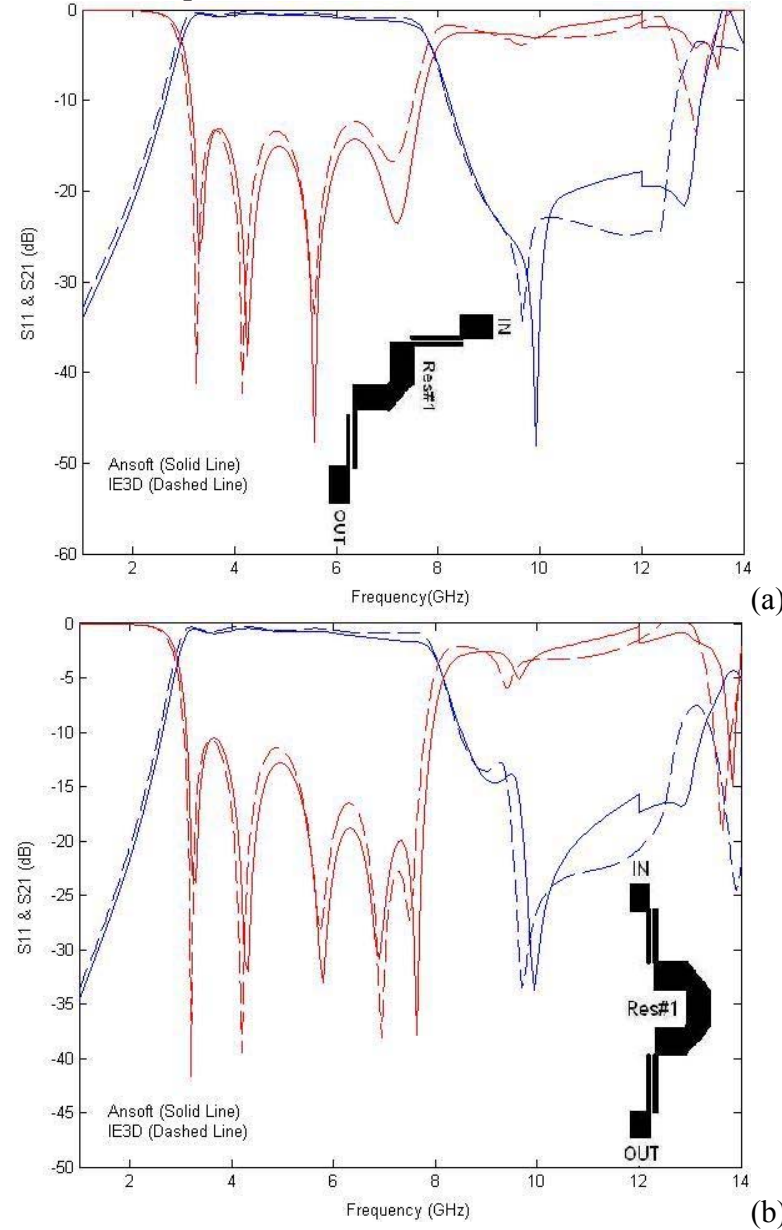

(a)

Fig. 3. Performances of single-SIR filters according to the folded configurations of Fig. 2b (a) and Fig. 2c (b). and the return loss is better than $11 \mathrm{~dB}$ for both filters, although the latter is slightly better for the filter in Fig. 3a. A transmission zero appears at $10 \mathrm{GHz}$ for both filters. The attenuation in the upper-band region for the filter in Fig. $3 \mathrm{~b}$ is slightly lower with an additional resonance peak at $9.5 \mathrm{GHz}$. This is due to the additional bend in the SIR, which produces unwanted coupling between resonant modes.

Fig. 4 shows the results of a single folded SIR filter according to Fig. 2a including source-load coupling. The design is for RT6006 substrate with a thickness of 25 mils. The symmetrical source-load coupling is positive and generates five additional transmission zeros - two on each side of the passband at $1.25,2.5,7.5$ and $8.4 \mathrm{GHz}$ as well as one in the upper-band region at $11 \mathrm{GHz}$. The 3-dB fractional bandwidth is 83 percent. The insertion loss is less than $1 \mathrm{~dB}$, and the return loss is better than $10 \mathrm{~dB}$.

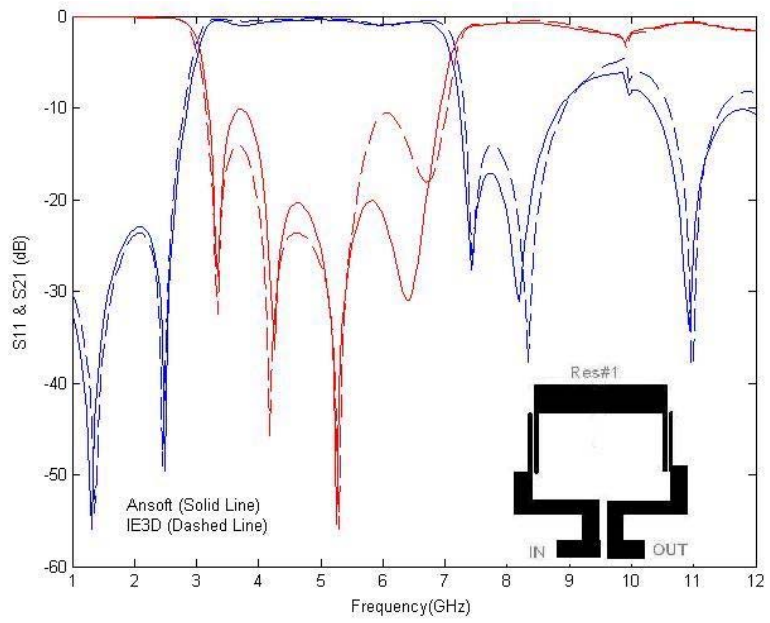

Fig. 4. Performance of single-SIR filter according to the folded configuration of Fig. 2a.

Fig. 5 shows results of SIR filters with two cascaded resonators according to the three folded configurations presented in Fig. 2. The structure in Fig. 5a utilizes RT6006 substrate $\left(\varepsilon_{\mathrm{r}}=6.15\right)$ with 25 mil thickness, those in Figs. 5b, 5c use RT6010 $\left(\varepsilon_{\mathrm{r}}=10.8\right)$ and 50 mils. The $3-\mathrm{dB}$ bandwidths in Figs. 5a, 5b, 5c are 4.7, 5.4 and $5.5 \mathrm{GHz}$, respectively. The insertion loss is less than $2 \mathrm{~dB}$, and the return loss is better than $10 \mathrm{~dB}$. Since source-load coupling is not in effect, no transmission zeros appear in the upper stopband in Fig. 5a; however, the attenuation is better than $30 \mathrm{~dB}$ from 8.5 to 14 GHz. Figs. 5b and 5c show some transmission zeros in the upper stopband. They are inherent to the folding of the individual SIR's as they have already been observed in the single-SIR structures in Fig. 3.

Fig. 6 shows the performances of two folded UWB filters with two cascaded SIR's and two different source-load coupling paths. The substrate used in these filters is RT6006 with 25 mil thickness. The source-load coupling generates altogether six real transmission zeros in the lower and upper rejection bands. It also creates a complex zero close to the upper side of the passband. Both filters have almost the same 
performance with an insertion loss less than $1 \mathrm{~dB}$ and return loss better than $15 \mathrm{~dB}$. The $3-\mathrm{dB}$ bandwidths are $4.7 \mathrm{GHz}$ centered at $5.45 \mathrm{GHz}$. It is noted that the locations of transmission zeros are the same for both filters; however, the filter in Fig. 6b is more compact. In comparison to Fig. 5a, the filters in Fig. 6 show no significant change in bandwidth and reflection coefficient (although the latter is slightly improved for the filters in Fig. 6), since the dimensions of the coupled-
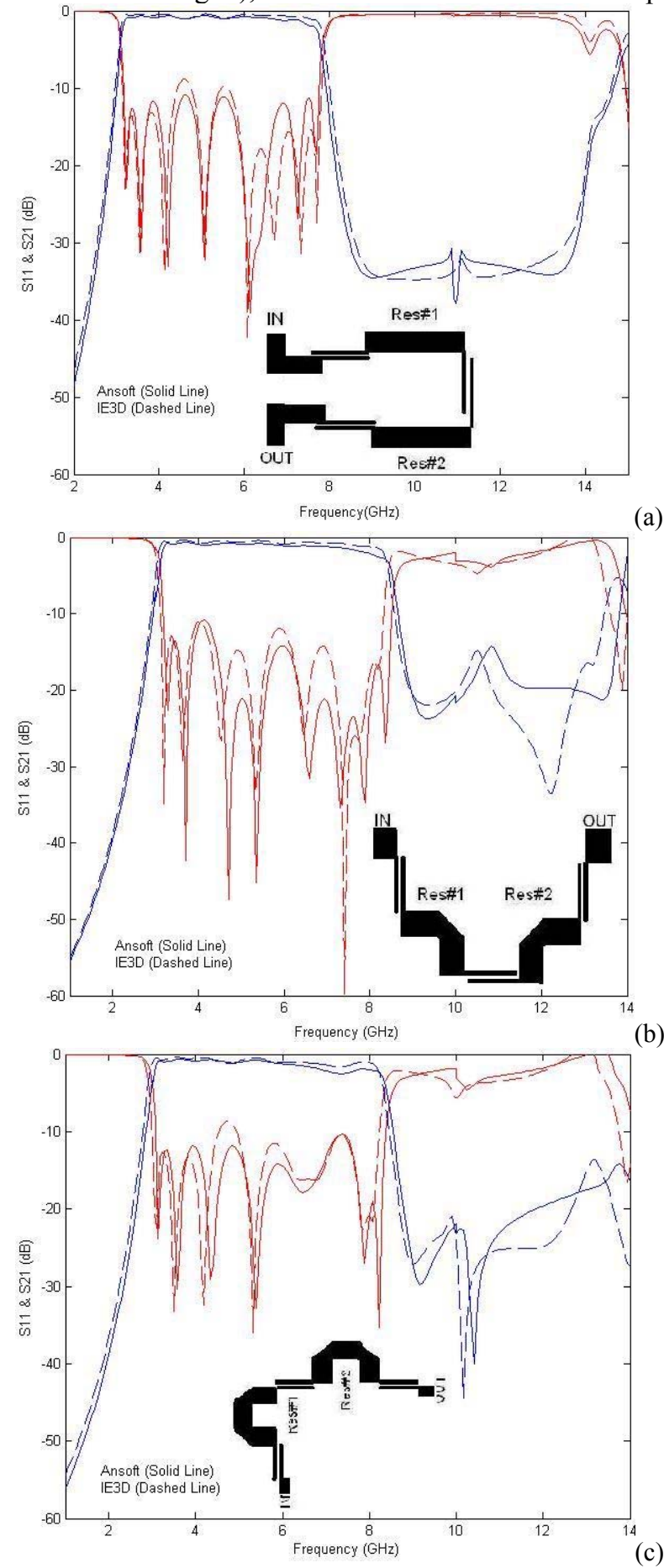

(c)

Fig. 5. Performances of two cascaded SIR filters according to the folded configurations of Figs. 2a (a), 2b (b) and 2c (c).

(a)

b) line sections and stepped-impedance resonators are kept unchanged. However, the source-load coupling in Fig. 6 has a great impact on the attenuation in the lower and upper rejection bands. This results in a high selectivity around the passband.
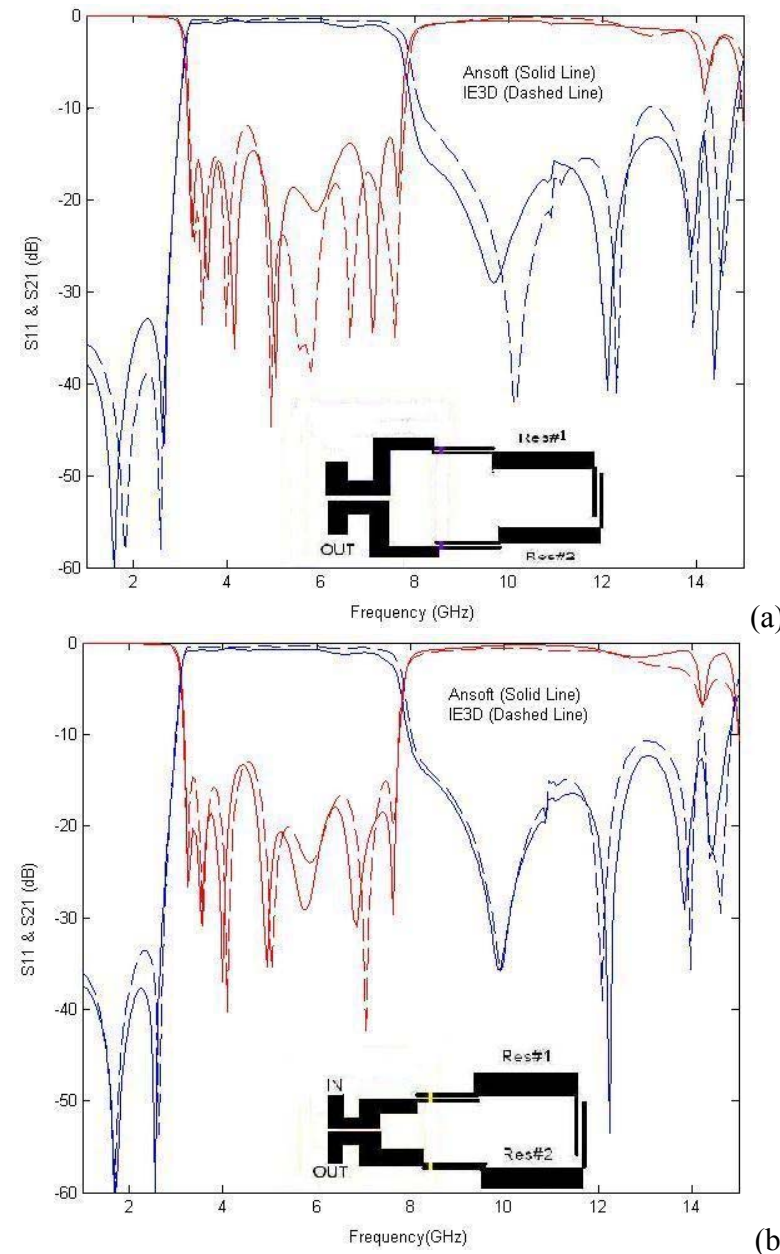

(a)

Fig. 6. Performances of two cascaded SIR filters according to Fig. 2a with source-load coupling: larger (a) and smaller (b) source-load coupling section.

Three cascaded SIR's are utilized to design an UWB filter to cover the entire 3.1-10.6 GHz band. Fig. 7 shows the results of UWB filters using three cascaded SIR's. The substrates used are 25-mil RT6006 in Fig. 7a and 50-mil RT6010 in Fig. 7b. The 3-dB fractional bandwidths are 104 and 102 percent in Figs. $7 \mathrm{a}$ and $7 \mathrm{~b}$, respectively. A transmission zero appears at the upper edge of the passband for both filters, but the attenuation is higher and over a wider stopband in Fig. 7b. Note that this performance is similar to that presented in [15], but the circuit in Fig. 7a is more compact due to the folded arrangement.

Fig. 8 shows the performance of the UWB filter in Fig. 7a, but with added source-load coupling. The attenuation in the upper stop-band region is $25 \mathrm{~dB}$ from 10 to $16 \mathrm{GHz}$. Five transmission zeros are generated through the source-load coupling. A complex zero also appears close to the upper edge 
of the passband at $10.5 \mathrm{GHz}$.
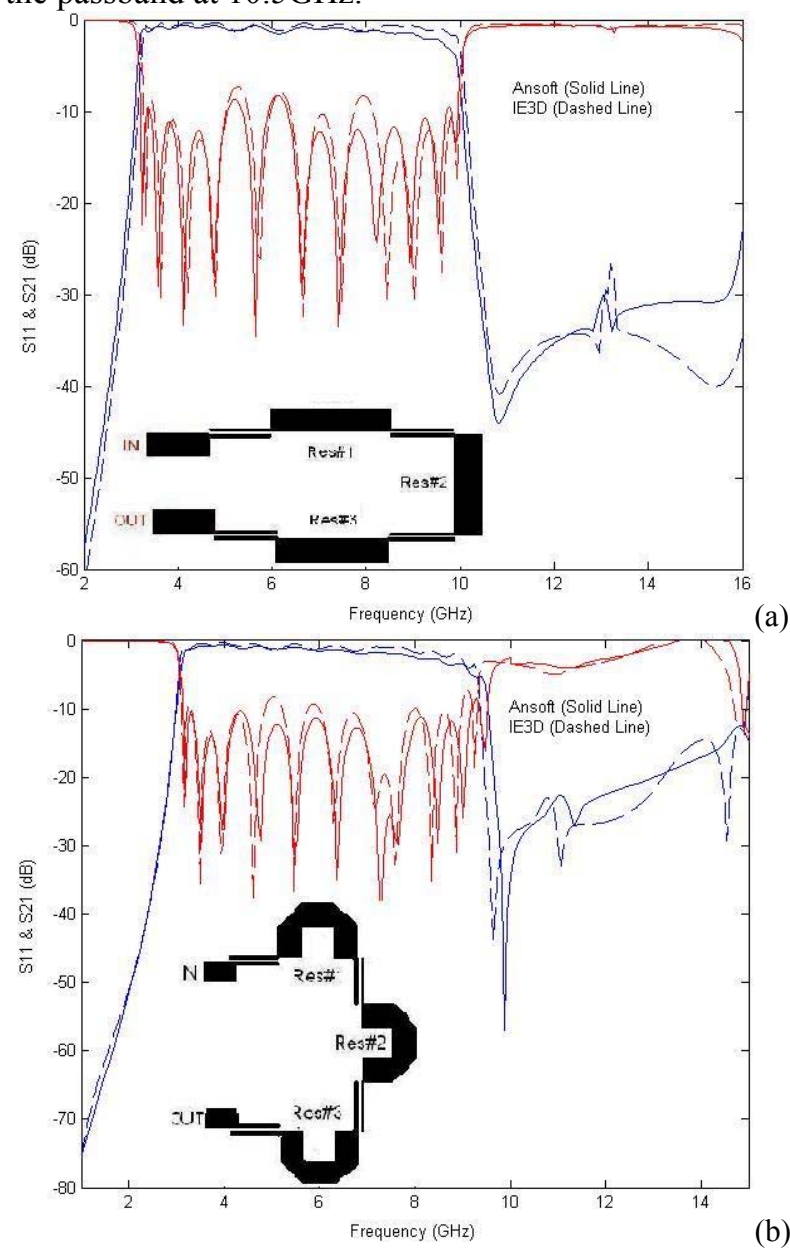

(a)

Fig. 7. Performances of two UWB filters using three cascaded SIR's according to Fig. 2a (a) and Fig. 2c (b).

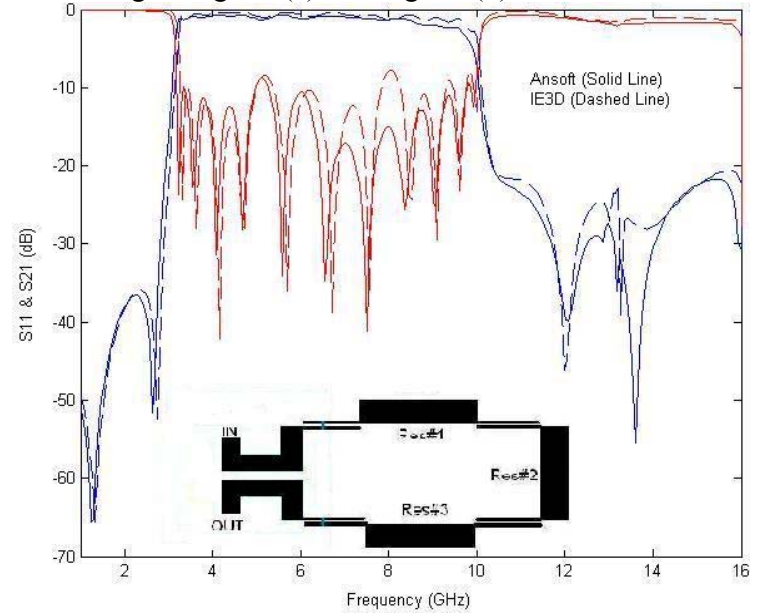

Fig. 8. Performance of UWB filter using three cascaded SIR's according to Fig. 2a and source-load coupling.

\section{CONCLUSIONS}

The different UWB filter designs utilizing folded steppedimpedance resonators have the following advantages: They combine compact size and improved performance due to structural folding of both the SIR's and the filter; they use planar microstrip technology and can be produced at relatively low cost; and they are well suited for ultra-wideband wireless applications.

\section{REFERENCES}

[1] J.-T. Kuo and H.-S. Cheng, "Design of quasi-elliptic function filters with a dual-passband response", IEEE Microwave Wireless Comp. Lett., Vol. 14, pp. 472-474, Oct. 2004.

[2] J.-T. Kuo, T.-H. Yeh and C.-C. Yeh, "Design of microstrip bandpass filters with a dual-passband response", IEEE Trans. Microwave Theory Tech., Vol. 53, pp. 1331-1337, Apr. 2005.

[3] S. Sun and L. Zhu, "Coupling dispersion of parallel-coupled microstrip lines for dual-band filters with controllable fractional pass bandwidths", in IEEE MTT-S Int. Microwave Symp. Dig., pp. 2195-2198, Long Beach, USA, June 2005.

[4] C.-M. Tsai, H.-M. Lee and C.-C. Tsai, "Planar filter design with fully controllable second passband", IEEE Trans. Microwave Theory Tech., Vol. 53, pp. 3429-3439, Nov. 2005.

[5] S. Sun and L. Zhu, "Novel design of dual-band microstrip bandpass filters with good in-between isolation", in Proc. AsiaPacific Microwave Conf., 4 pp., Suzhou, China, Dec. 2005.

[6] T.-H. Huang, H.-J. Chen, C.-S. Chang, L.-S. Chen, Y.-H. Wang and M.-P. Houng, "A novel compact ring dual-mode filter with adjustable second-passband for dual-band applications", IEEE Microwave Wireless Comp. Lett., Vol. 16, pp. 360-362, June 2006.

[7] M. Mokhtaari, J. Bornemann and S. Amari, "New reduced-size step-impedance dual-band filters with enhanced bandwidth and stopband performance", in 2006 IEEE MTT-S Int. Microwave Symp. Dig., pp. 1181-1184, San Francisco, USA, June 2006.

[8] J. Wang, Y.-X. Guo, B.Z. Wang and L.C. Ong, "Dual-band stepped-impedance band-pass filter", in Proc. 36th European Microwave Conf., pp. 902-904, Manchester, UK, Oct. 2006.

[9] H. Wang, L. Zhu and W. Menzel, "Ultra-wideband bandpass filter with hybrid microstrip/CPW structure", IEEE Microwave Wireless Comp. Lett., Vol. 15, pp. 844-846, Dec. 2005.

[10] K. Li, "UWB bandpass filter: Structure, performance and application to UWB pulse generation", Proc. 2005 Asia-Pacific Microwave Conf., pp. 79-82, Suzhou, China, Dec. 2005.

[11] J. Gao, L. Zhu, W. Menze and F. Bögelsack, "Short-circuited CPW multiple-mode resonator for ultra-wideband (UWB) bandpass filter", IEEE Microwave Wireless Comp. Lett., Vol. 16, pp. 104-106, Mar. 2006.

[12] W. Menzel, L. Zhu, K. Wu and F. Bögelsack, "On the design of novel compact broad-band planar filters", IEEE Trans. Microwave Theory Tech., Vol. 51, pp. 364-370, Feb. 2003.

[13] L. Zhu, S. Sun and W. Menzel, "Ultra-wideband (UWB) bandpass filters using multiple-mode resonator", IEEE Microwave Wireless Comp. Lett., Vol. 15, pp. 796-798, Nov. 2005.

[14] M. Mokhtaari, J. Bornemann and S. Amari, "Compact planar ultra-wide pass-band filters with source-load coupling and impedance stubs", Proc. 2006 Asia-Pacific Microwave Conf., Yokohama, Japan, Dec. 2006.

[15] Y.-C. Chiou, J.-T. Kuo, E. Chang, "Broadband quasiChebyshev bandpass filter with multimode stepped-impedance resonators (SIRs)", IEEE Trans. Microwave Theory Tech., Vol. 54, pp. 3352-3358, Aug. 2006. 